\title{
Modelling and dynamic performance of inverter based generation in power system studies: an international questionnaire survey
}

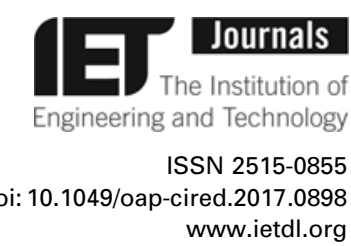

www.ietdl.org

\author{
Gustav Lammert ${ }^{1 凶}$, Koji Yamashita ${ }^{2}$, Luis David Pabón Ospina ${ }^{3}$, \\ Herwig Renner ${ }^{4}$, Sergio Martínez Villanueva ${ }^{5}$, Pouyan Pourbeik ${ }^{6}$, \\ Florin-Emilian Ciausiu7, Martin Braun 1,3 \\ ${ }^{1}$ University of Kassel, Kassel, Germany \\ ${ }^{2}$ CRIEPI, Kanagawa, Japan \\ ${ }^{3}$ Fraunhofer IWES, Kassel, Germany \\ ${ }^{4}$ Graz University of Technology, Graz, Austria \\ ${ }^{5}$ Red Eléctrica de España, Alcobendas, Spain \\ ${ }^{6}$ PEACE-PLLC, Flower Mound, USA \\ ${ }^{7}$ Tractebel ENGIE, Bucharest, Romania \\ $\bowtie$ E-mail: gustav.lammert@uni-kassel.de
}

\begin{abstract}
Owing to the increasing penetration level of Inverter Based Generation (IBG) over the last years, there has been much effort on the development of IBG models for power system dynamic studies. Therefore, CIGRE and CIRED have established a joint working group CIGRE C4/C6.35/CIRED: 'Modelling and dynamic performance of inverter based generation in power system transmission and distribution studies' with the aim of collecting the present best practices in the industry on modelling of IBG for power system dynamic studies, with the focus on PhotoVoltaic (PV) systems. For this purpose, a questionnaire was developed and distributed to utilities and system operators around the world. This paper summarises some of the key findings and observations and identifies prevalent information about: (i) the type of IBG technology that is modelled (e.g. wind, PV etc.); (ii) the used IBG models (individual or aggregated models); and (iii) the type of the power system dynamic study performed. This survey supports utilities and system operators as well as research institutes and academia to benchmark their approach against the prevailing international industry practice.
\end{abstract}

\section{Introduction}

Nowadays, the electrical power system is undergoing fundamental changes due to the ever increasing penetration of Inverter Based Generation (IBG). The dynamic characteristic of IBG, such as wind or PhotoVoltaic (PV) generation, is fundamentally different from the conventional synchronous generators, and thus the dynamic performance of the system following disturbances might change due to the increasing impact of IBG. Therefore, studies are required that analyse the impact of high penetration levels of IBG on the dynamic performance of the system. In this context, the selection of the IBG model type is crucial for the analysis.

There are many levels of models used for all types of power system components. On the one hand, there are detailed models of IBG, also referred to as individual models. On the other hand, there are simplified models that represent an aggregation of IBG, also referred to as aggregated models. However, since all models have limitations, the selection of the model type is crucial based on the objectives of the study.

Different types of models for IBG have been developed over the last years. Two industry working groups were established, one within the Western Electricity Coordinating Council (WECC) [1], and the other within the International Electrotechnical Commission (IEC) [2], in order to develop generic models of different types of IBG. Some of those models have been already implemented in widely used commercial power system analysis software tools [3, 4].
However, these generic models are still not widely used yet by the industry.

In 2013, the new Joint Working Group (JWG) CIGRE C4/C6.35/ CIRED: 'Modelling and dynamic performance of inverter based generation in power system transmission and distribution studies' was established, to look at some of these evolving issues. One of the tasks of the JWG is to identify the present industry practice on modelling of IBG, with the focus on PV generation. For that purpose, a comprehensive questionnaire was distributed to utilities and system operators around the world. This paper summarises some of the key findings.

The aim of this paper is not necessarily to recommend the application of any specific dynamic model for a specific power system dynamic study, but, rather, to identify what dynamic models are presently applied and provide some real pictures on their application.

\section{Survey}

\subsection{Organisation}

The original questionnaire that was distributed to the utilities and system operators consists of four categories, whereas this paper focuses on the results of two of them. The two categories and the corresponding questions to each of them are listed in Table 1. Further findings and observations of the original questionnaire can be found in [5]. 
Table 1 Survey categories and questions

\begin{tabular}{|c|c|c|c|}
\hline No. & Category & No. & Question \\
\hline 1 & type of the IBG technology and its modelling & $\begin{array}{l}1.1 \\
1.2 \\
1.3 \\
1.4 \\
1.5\end{array}$ & $\begin{array}{l}\text { Which of the following IBG technologies do you model for power system } \\
\text { dynamic studies? } \\
\text { wind } \\
\text { PV } \\
\text { micro turbine } \\
\text { fuel cell } \\
\text { battery energy system }\end{array}$ \\
\hline
\end{tabular}

a Voltage fluctuations at steady state are not considered as long-term voltage stability.

Category 1 sorts the IBG technology and its modelling by type. The participants of the survey could select between different technologies, such as wind or PV generation, micro turbines, fuel cells or battery energy systems. This category reveals the type of IBG technology modelled by utilities and system operators for power system dynamic studies.

Category 2 describes the type of model that is currently used by the utility and system operator for a specific type of power system dynamic study. In this category, two types of models are classified, namely individual models and aggregated models. A detailed comparison between individual and aggregated models is provided in Section 3. Furthermore, category 2 refers to 14 different types of power system dynamic studies, including well-known phenomena such as frequency stability, short-term and long-term voltage stability as well as transient and small-disturbance angle stability.

\subsection{Participants}

Potential survey participants were selected by the JWG members and contacted via e-mail. The considered utilities and system operators were either Transmission System Operators (TSOs), or utilities and system operators that operate both the transmission and distribution systems. However, Distribution System Operators (DSOs) were not included in the results of this paper, because the responses show that power system dynamic studies are barely conducted by DSOs.

The developed questionnaire was sent to 63 utilities and system operators around the world between spring 2015 and summer 2016. Out of these 63 contacted utilities and system operators, 45 replied to the JWG. Hence, the response rate of $71 \%$ was reached. The 45 questionnaires received from utilities and system operators came from 21 countries on five continents. The participants mostly operate an interconnected grid, and only a few operate an isolated small grid. The system sizes vary from small-scale power systems (a few megawatts) to large-scale power systems (several gigawatts).

It should be noted that software vendors, consultancies, research organisations and academia are not included in the interviewees. Out of the 45 questionnaires received, 35 came from TSOs, and 10 came from utilities and system operators that operate both the transmission and distribution systems.

\section{Type of models}

Usually, power system dynamic studies are examined in various system sizes, such as studies in a local power system or a bulk power system. Therefore, various types of models are applied. In general, there are many levels of models used for all types of IBG. This paper focuses on individual and aggregated models of IBG, as explained in the following subsections.

\subsection{Individual models}

In this paper, individual models of IBG are defined as: (i) one model for each generator/inverter; or (ii) one model for a group of generators/inverters within the same power plant/park/farm. For a dynamic study in a local power system, an individual model is more likely to be applied.

3.1.1 Individual models of large-scale power plants of IBG: Individual models of large-scale power plants which consist of a group of generators/inverters are not likely to be modelled individually even for power system dynamic studies in the local network. As not only the generator/inverter type but also the manufacturer can be the same, the single-machine equivalent model, as recommended by e.g. WECC [6], can be used for local power system dynamic studies. Therefore, the IBG plant is represented by the collector system equivalent, the step-up transformer equivalent and the generator/inverter equivalent [7], as shown in Fig. 1. In addition, this model may also be used for bulk power system studies.

3.1.2 Individual models of small-scale distributed systems of IBG: If the system is not too large, individual models of small-scale distributed systems may explicitly represent each generator/inverter, load, line etc. (the distribution system) individually for the local power system dynamic study, as depicted in Fig. 2. It should be noted that the structure of the network in Fig. 2 is just an example.

\subsection{Aggregated models}

In this paper, aggregated models of IBG are defined as the lumped model, which represents the dynamic behaviour of the system in an aggregated manner. For a dynamic study in a bulk power system, an aggregated model is more likely to be applied. 


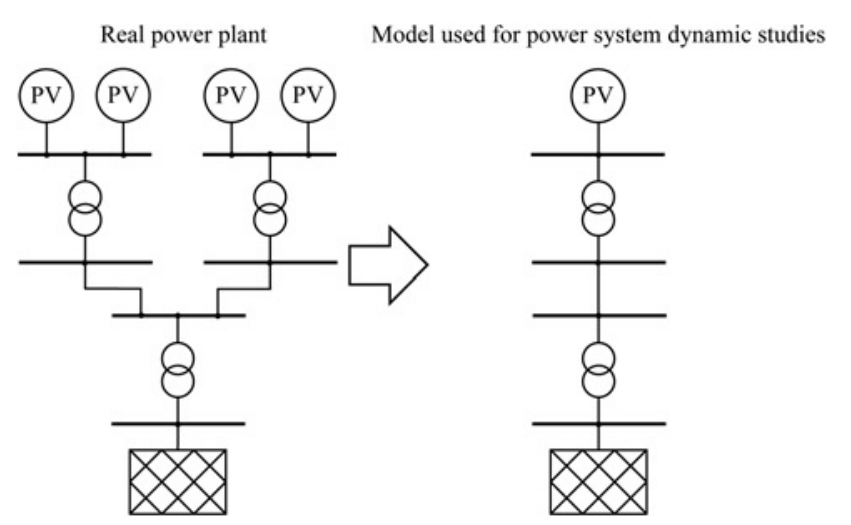

Fig. 1 Individual model of a large-scale PV plant (adapted from WECC [6])

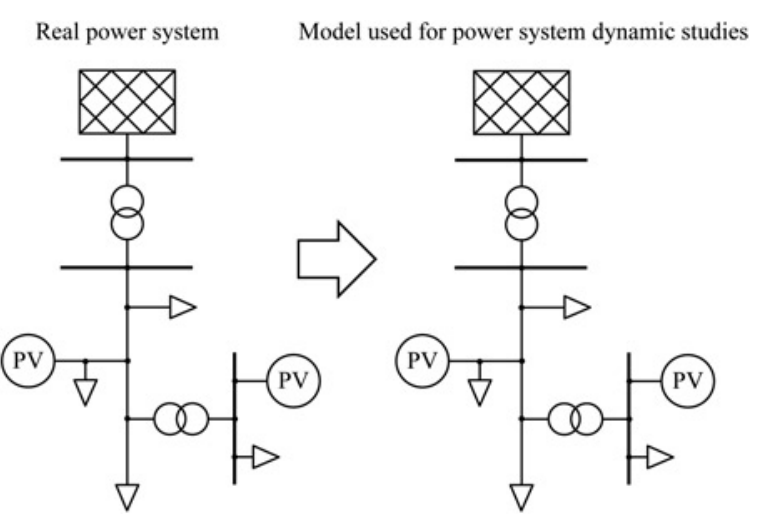

Fig. 2 Individual model of small-scale distributed PV systems

3.2.1 Aggregated models of small-scale distributed systems of IBG: Small-scale distributed systems of IBG e.g. residential PV can be represented as an aggregated model, as recommended by e.g. WECC [6] for bulk power system dynamic studies. Therefore, the distribution system is modelled with the equivalent impedance, the load equivalent and the generator/ inverter equivalent, as illustrated in Fig. 3. The goal is to capture the impact of small-scale distributed IBG, lumped at the transmission level, on the bulk system performance. However, there are still no widely accepted aggregated dynamic models for small-scale distributed IBG. In this context, the most recent work has been done by WECC [8], but it is currently under review and discussion for further changes.

\section{Results}

\subsection{Category 1: type of the IBG technology and its modelling}

Category 1 is about the predominant IBG technologies that are modelled by utilities and system operators and the results are shown in Fig. 4. It can be concluded that wind and PV generation are predominantly modelled for power system dynamic studies with 76 and $67 \%$, respectively. Furthermore, $24 \%$ of the participants model battery energy systems, and only 9 and $7 \%$ model micro turbines and fuel cells, respectively.

\subsection{Category 2: type of model used for a specific type of power system dynamic study}

Category 2 is about the type of model that is used by utilities and system operators for a specific type of power system dynamic

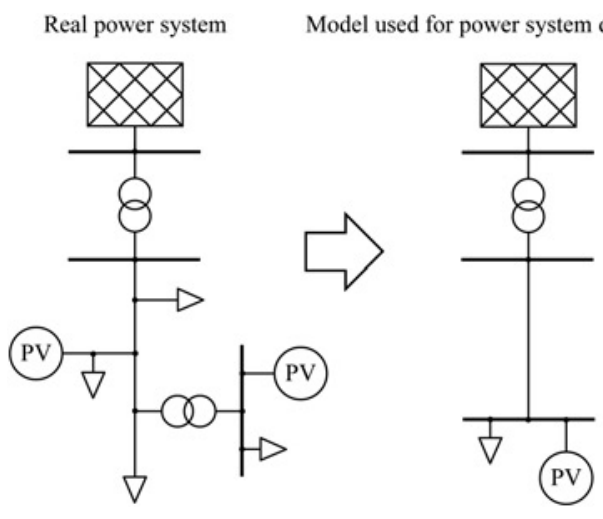

Fig. 3 Aggregated model of small-scale distributed PV systems (adapted from WECC [6])

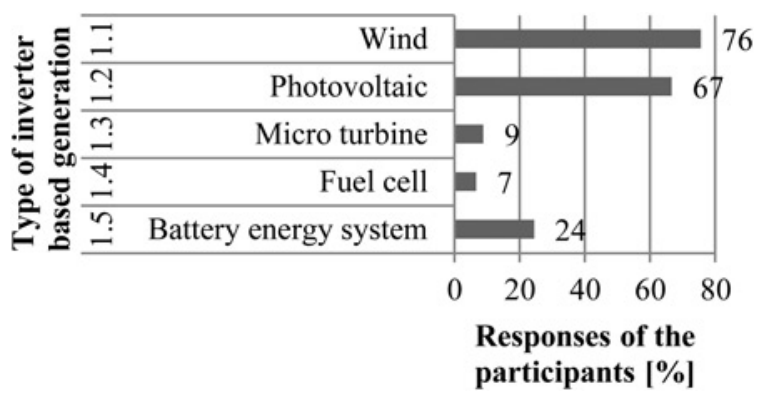

Fig. 4 Question 1: Which of the following IBG technologies do you model for power system dynamic studies?

study and the results are depicted in Fig. 5. In general, it can be seen that the application of individual models for IBG is slightly higher than the application of aggregated models, and reaches 59\% in average, as indicated in the last row of the figure.

The share of aggregated models is slightly higher for high-voltage ride-through and transient stability studies, compared to the average value. These results are identical to the aforementioned observations because those dynamic studies are typically performed in a bulk power system.

The share of individual models is slightly higher for voltage stability and control system interactions studies, compared to the average value. These results are identical to the aforementioned observations because those dynamic studies are typically performed in a local power system.

\section{Conclusions}

The aim of this paper is not necessarily to recommend the application of any dynamic model for a specific type of power system dynamic study, but, rather, to identify what dynamic models are currently applied and provide some fundamental information on their use.

The main contributions and key findings of this paper can be summarised as follows:

- Wind and PV generation is very likely to be modelled by utilities and system operators for power system dynamic studies with 76 and $67 \%$, respectively.

- Overall $59 \%$ of the utilities and system operators apply individual models instead of aggregated models for power system dynamic studies.

In general, an individual model for IBG is typically used for the analysis in the local power system of e.g. protection coordination, 


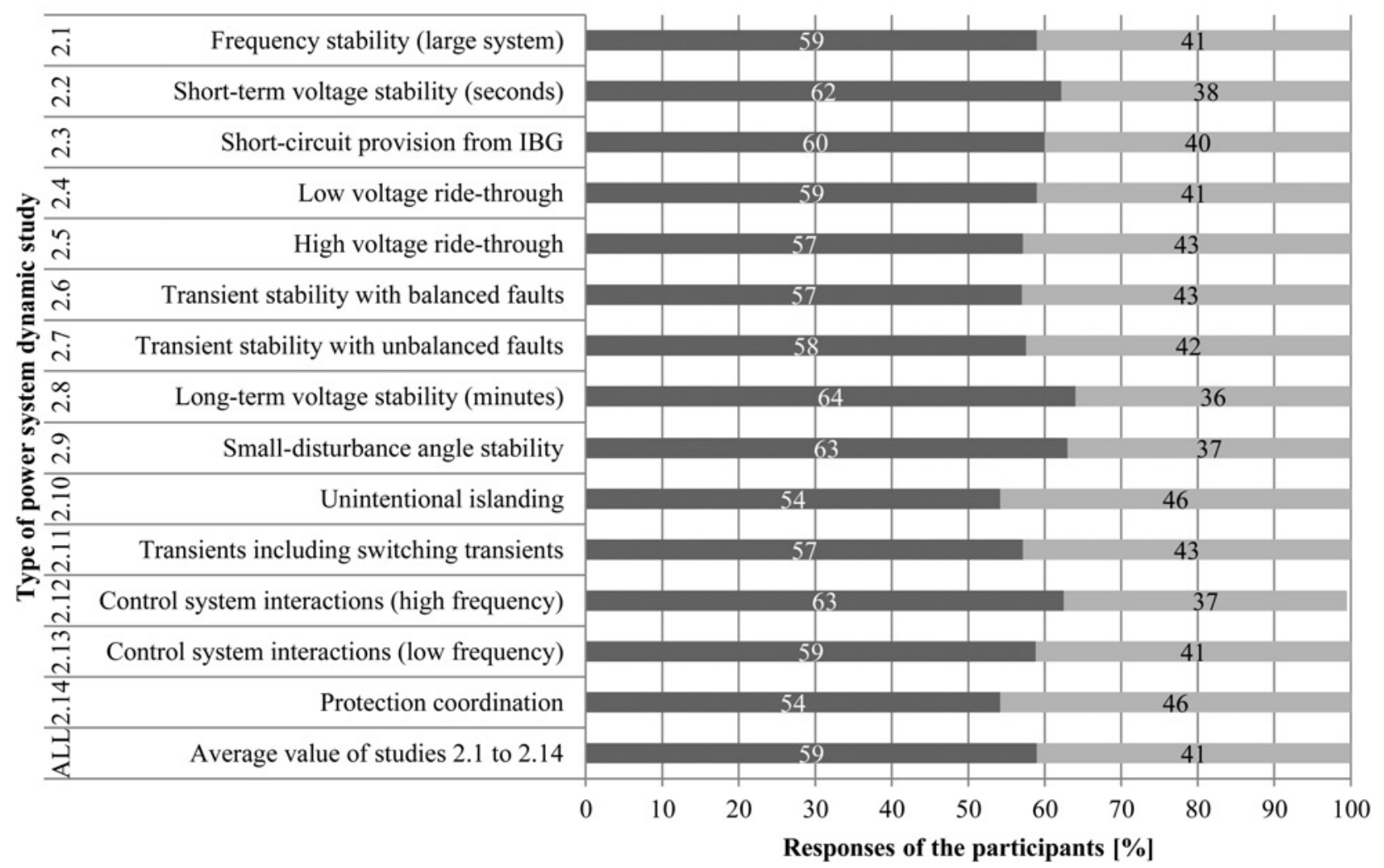

Fig. 5 Question 2: What type of model do you use for a specific type of power system dynamic study?

unintentional islanding, short-circuit provision from IBG, transients including switching transients, long-term and short-term voltage stabilities and control system interactions (low and high frequency).

On the other hand, an aggregated model for IBG is typically used for the analysis in the bulk power system of e.g. frequency stability, low-voltage and high-voltage ride-through, transient stability (with balanced and unbalanced faults) and small-disturbance angle stability.

However, for specific power system dynamic studies the aforementioned application of the different types of models might also be used vice versa.

It can be concluded that every type of model has advantages and disadvantages and the adequate model type needs to be selected depending on the type of power system dynamic study and the power system condition (e.g. weak system conditions with very low short-circuit ratio or strong system conditions with high short-circuit ratio).

The results of the questionnaire emphasise the clear message for the necessity and importance of the use of IBG models. Furthermore, the final technical brochure of the CIGRE JWG C4/ C6.35/CIRED will give guidance in selecting adequate models for IBG for specific power system dynamic studies.

With these contributions the paper supports utilities and system operators as well as research institutes and academia to benchmark their approach against the prevailing international industry practice.

\section{Acknowledgments}

The authors appreciate the support of their colleagues from the CIGRE JWG C4/C6.35/CIRED on 'Modelling and dynamic performance of inverter based generation in power system transmission and distribution studies', who contributed in many different, but always useful ways to this paper. Equally important, the authors would like to acknowledge the support of the colleagues from utilities and system operators around the world, who took the time to fill in the questionnaire and kindly shared their knowledge and experience. The work of some of the authors was supported by the German Federal Ministry for Economic Affairs and Energy and the Projektträger Jülich GmbH (PTJ) within the framework of the project Smart Grid Models (FKZ: 0325616) and DEA-Stabil (FKZ: 0325585A).

\section{References}

1 Western Electricity Coordinating Council (WECC) Renewable Energy Modeling Task Force

2 International Electrotechnical Commission (IEC) TC88 WG27, 'Wind turbines electrical simulation models for wind power generation'

3 Elliott, R., Ellis, A., Pourbeik, P., et al.: 'Generic photovoltaic system models for WECC - a status report'. 2015 IEEE Power \& Energy Society General Meeting, Denver, July 2015 , pp. 1-5

4 Lammert, G., Pabón Ospina, L.D., Pourbeik, P., et al.: 'Implementation and validation of WECC generic photovoltaic system models in DIgSILENT PowerFactory'. 2016 IEEE Power \& Energy Society General Meeting, Boston, July 2016, pp. 1-5

5 Lammert, G., Yamashita, K., Pabón Ospina, L.D., et al.: 'International industry practice on modelling and dynamic performance of inverter based generation in power system studies'. CIGRE Science \& Engineering, 2017, 8, pp. 25-37

6 Western Electricity Coordinating Council (WECC) Renewable Energy Modeling Task Force: 'WECC PV plant power flow modeling guidelines', August 2010, pp. $1-15$

7 Soni, S., Karady, G.G., Morjaria, M., et al.: 'Comparison of full and reduced scale solar PV plant models in multi-machine power systems'. 2014 IEEE PES T\&D Conf. and Exposition, Chicago, April 2014, pp. 1-5

8 Pourbeik, P.: 'Proposal for the DER_A model'. Presentation at WECC Meeting, November 2016 